\title{
A Non Invasive Biomarkers for Alzheimer's disease Detection
}

\author{
Ramya. M, Marykutty Cyriac
}

\begin{abstract}
Alzheimer's disease (AD) is one of the most common neurodegenerative diseases occurring in elderly population worldwide, which usually starts slowly and worsens over time. AD is generally diagnosed too late, when irreversible damages have been caused in the patient's brain region. Present need demands the discovery of diagnostic and prognostic patient specific effective biomarkers to improve patient's life quality and avoid big healthcare costs. Objective of this survey is to review the non-invasive biomarkers that could be used to predict early onset of $A D$ and delay cognitive impairment.
\end{abstract}

Index Terms- Alzheimer's disease, biomarkers, cognitive impairment, neuro-degenerative disease.

\section{INTRODUCTION}

Alzheimer's disease (AD) is an age related neurodegenerative disorder that leads to severe cognitive loss and eventually death in the elderly population of 60 years and above. According to the current reports, it is estimated that, by 2050, one new case of AD will develop in every 33 seconds and the total expected occurrence will be 13.8 million around the world [1]. As the AD progresses, Neurofibrillary tangles (NFTs) and amyloid- $\beta$ (A $\beta$ ) plaque deposition occurs in the brain, followed by atrophy in structures such as hippocampus region and finally degeneration of the whole brain. Besides age, a few other risk factors leading to AD are smoking, diabetes, hypertension, lifestyle and stress. Depression, lack of social activities and head injury are other risk factors for the AD.

An early symptom of the AD is difficulty in remembering the recent events namely, short-term memory loss. Other symptoms on disease progression are problems with language, disorientation, mood swings, loss of motivation, inability of self-care and behavioural issues. Early detection and treatment of $\mathrm{AD}$ could reduce the brain damage that generally occurs in $\mathrm{AD}$ patients. One of the most promising methods for early detection of the $\mathrm{AD}$ is using biomarkers. The term biomarker or biological marker is the objective indication of medical state observed from outside the patients, which can be measured accurately [2]. A Biomarker is basically defined as any substance, structure or process that can be measured inside the body or in its products and influence any changes in the body or predicts the outcome or disease [3]. In general, biomarkers used for the detection of $\mathrm{AD}$ could be either invasive or non-invasive.

Revised Manuscript Received on December 22, 2018.

Ramya.M, Research Scholar, Electronics and Communication Engineering, Jerusalem College of Engineering, Anna University, Chennai, India.(e-mail: ramshems@gmail.com).

Dr.Marykutty Cyriac, Professor, Electronics and Communication Engineering, Jerusalem College of Engineering, Chennai, India.(e-mail:marycyriac123@gmail.com).
A major invasive biomarker is the CSF fluid, as it replicates metabolic processes in the brain. However, it has limited use because of its invasive nature. Recent advancements in the medical signal processing and imaging technologies have led to the development of non-invasive biomarkers. Non-invasive biomarkers can be classified as Imaging biomarkers, Signal biomarkers and olfactory dysfunction biomarkers. The objective of this paper is to review the non-invasive biomarkers and list out their features.

\section{IMAGING BIOMARKERS}

Imaging Biomarkers are diagnostically relevant features that are extracted from medical images through image processing techniques. Out of various possible biomarkers, neuro-imaging biomarkers are having high potential in the prediction of AD. Neuro-imaging methods fall into two categories; structural and functional. Structural imaging methods are CT and MRI. CT is a less preferred method for early diagnosis of $\mathrm{AD}$. MRI is the most commonly used structural imaging technique for $\mathrm{AD}$ brains. Functional imaging techniques such as fMRI and SPECT have less spatial resolution than the structural methods

\section{A Neuro-Imaging Biomarkers}

Imaging modalities used to identify biomarkers in the brain region for the $\mathrm{AD}$ diagnosis are Magnetic Resonance imaging (MRI), functional magnetic resonance imaging (fMRI), fluorodeoxyglucose-positron Emission tomography (FDG-PET), Magnetic Resonance Spectroscopy (MRS) and DTI. For AD classification using MRI data, relationship induced multi-template learning (RIML) method was proposed in [4]. Affinity Propagation (AP) clustering algorithm was used to partition the entire population (i.e., $\mathrm{AD}$ and $\mathrm{NC}$ brain images) into 10 non-overlapping clusters and ten templates were determined from these clusters Performance of the Relationship induced sparse (RIS) feature selection algorithm was compared with the Pearson, COMPARE, T-test and Lasso feature selection algorithm using single template and multi template data. Ensemble classification method was developed and used to combine the outputs of multiple SVMs for final classification. The Alzheimer's Disease Neuroimaging Initiative (ADNI) database was used to conduct the experiments. Experimental results demonstrated significant classification accuracy in the AD classification. In another approach, a whole brain hierarchical network was developed to extract features for MRI-based classification of AD. Spatial-correlations were used to extract texture features from the Regions of

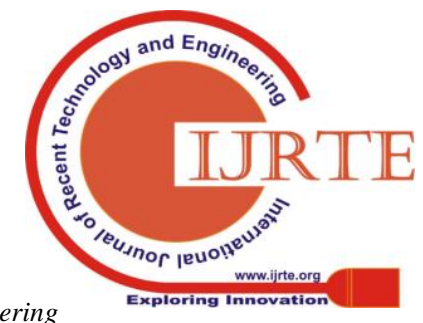


interest (ROIs). Multiple kernel classifier (MKBoost) was used to classify Health controls (HC), MCI, MCI converted to $\mathrm{AD}$ (MCIc), MCI not converted to $\mathrm{AD}$ (MCInc) and $\mathrm{AD}$ [5]. In another study, multiple magnetic resonance features such as hippocampal volume, tensor-based morphometry, cortical thickness and a novel technique based on manifold learning were used to classify images. An improvement in classification was obtained with linear discriminant analysis (LDA) than support vector machines classifier [6]. A statistical, automated brain MRI diagnostic system was developed in [7] with a two-stage algorithm for normal and (AD) MRIs differentiation. After pre-processing steps with the morphological and low pass filter, intensity histogram was computed for each image and mean and standard deviation were calculated.

A method using Discrete Wavelet Transform (DWT) for feature extraction, Principal Component Analysis (PCA) for feature reduction, and Normalized Mutual Information Feature Selection (NMIFS) algorithm for feature selection was proposed in [8]. SVM classifier was used for the classification. It was found that the use of dimensionality reduction led to a reduced classification accuracy. A methodology for computer-aided diagnosis was presented and demonstrated using simulated and real data, that it appears robust to small variations in MRI acquisition parameters. The results showed that SVMs are marginally superior to LDA and quadratic discriminant analysis (QDA) classification techniques. The MR-based calculation could cut the diagnosis error by a factor of two. This could have profound implication in managing the suspected $\mathrm{AD}$ candidates [9]. A diffusion model with impulsive sources over the brain connectivity network was suggested to model the progress of brain atrophy. Atrophy sources were reliably estimated by imposing the sparse regularization on the source distribution. An efficient gradient descent method was adopted to solve the inverse problem of tracking back the diffusion sources. The changes of brain atrophy patterns were effectively predicted by this model- Possible origins and progression of the diseases in an early stage was easily understood by using longitudinal MRI data in this diffusion model [10].

Machine learning tools were employed to detect the selectively affected brain regions using the data gathered in a fMRI study of demented and non-demented patients. For analysing these kinds of data, SVM-based classifier (sequential minimal optimization-SMO) and voxel selector (Random feature elimination-RFE) was highlighted as the best-suited data mining approaches [11]. Optimally-discriminative voxel-based analysis (ODVBA) frame work was proposed to precisely describe the shape and location of structural abnormality in the brain region of the MRI images. Classification rate was determined with ODVBA than traditional statistical parametric mapping (SPM) and the nonparametric SPM (SnPM) in [12].

A novel regularized, clustering method CHIMERA [13] was proposed to analyse the heterogeneity of $\mathrm{AD}$. Two patient groups, corresponding to distinct pathological brain atrophy patterns were recognized by CHIMERA using structural MRI of $\mathrm{AD}$ patients. MMSE score, Alzheimer's Disease Assessment Scale-Cognitive Subscale (ADAS-cog) 11 score and ADAS-cog 13 score were used to analyse the clinical cognitive performance of the two sub groups. Early and late onset $\mathrm{AD}$ subtypes which displays significant differences in cognitive performance were discovered by CHIMERA. Compared to $\mathrm{K}$-means and hierarchical Ward clustering improved clustering results were produced by CHIMERA. Healthy, aged and typical AD brains with different characterization of brain volumetric changes were modelled based on time-resolved 3-D Monte Carlo simulation from MRI data. In the results, the important difference of prefrontal cortex structure could be observed among aged, healthy, and AD brain with various source-detector separations in Sagittal view. The AD brain has larger CSF and smaller grey matter (GM) and white matter (WM) than the other cases were identified in this proposed work [14].

A landmark-based feature extraction method [15] which is 50 times faster was proposed for AD classification. Time consuming approaches like nonlinear registration and tissue segmentation were not used in this work. AD landmarks capable of differentiating $\mathrm{AD}$ subjects from $\mathrm{HCs}$ were identified using an efficient technique based on a shape-constrained regression-forest algorithm in the training stage. Detection accuracy can be improved by identifying the extra set of salient and consistent landmarks. SVM classifier that is capable of predicting the $\mathrm{AD}$ condition was trained using the identified landmarks .The performance of the SVM classifier is comparable to, or even better than that achieved by existing region-based and voxel-based methods.

The Structural MRI markers support earlier and more-precise diagnosis and measurement of progression of $\mathrm{AD}$. The existence of atrophy of medial temporal structures was a partially validated candidate marker for early diagnosis of the disease at the MCI stage. Rates of whole-brain and hippocampal atrophy were sensitive and powerful markers of progression of neurodegenerative disease. Structural imaging changes were present at the crossroads between the molecular pathology of $\mathrm{AD}$ and the clinical and cognitive decline that follow from that pathology. Structural imaging contributes to improved early diagnosis of $\mathrm{AD}$ and to the search for treatments to slow or prevent this devastating disease [16].

An inherent structure-based multi view learning (ISML) method was developed with feature representation generated from multiple templates for AD/MCI classification. Specific SVM classifier was learned using selected features in each view space. Results of multiple SVMs were fused together by a majority voting strategy. The efficacy of the proposed method was evaluated on a cohort of subjects with MRI baseline data, Mini-Mental State Examination (MMSE) and Clinical Dementia Rating (CDR) from the ADNI database. Better accuracies for $\mathrm{AD}$ versus normal controls (NC), progressive MCI (pMCI) versus NC, and pMCI versus stable MCI (sMCI) classification tasks were obtained in [17].

A unique sparse learning method called temporally constrained group least absolute shrinkage and selection operator (tgLASSO) was recommended for longitudinal analysis of data with multiple time points.

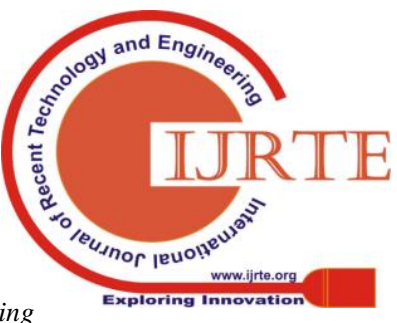
\& Sciences Publication 
MRI data and clinical scores including MMSE and ADAS-Cog were used as the multimodal input data. An efficient iterative optimization algorithm was improved for solving the new objective function. Improvement in Regression performance and discovery of the disease related imaging biomarkers were achieved in [18].

Hippocampal texture had a considerably higher differentiation between stable MCIs and MCI-to-AD converters within 24 months than hippocampal volume. A bilateral hippocampal texture score was computed by combining a texture descriptor and a SVM. Both texture and volume correlated with MMSE. In contrast, only texture correlated with Addenbrooke's cognitive Examination (ACE). In addition, a significant correlation between texture and hippocampal fluorodeoxy glucose-positron Emission tomography (FDG-PET) uptake was found. And the combined texture and volume did not significantly improve diagnostic or prognostic area under the receiver operating characteristic curve (AUC) compared to texture alone [19].

The proposed method TANNN [20] merges Statistical and Data comparisons classification methods. Very high performance was achieved when compared with other classification techniques such as K-Nearest Neighbour algorithm (KNN), SVM, Decision Tree (DT), and Naïve Bayes. Relatively fast classification and high detection rate could be achieved with this technique. This method is best suited for real-time classification tasks. The MR images and Magnetic Resonance Spectroscopy (MRS) of the three real Alzheimer datasets was analysed and results indicated that the patient Prevalence of AD doubles every 5 years of life after age 60 .

A discriminative sparse learning framework with multi relation regularization was used for feature selection of MRI and positron emission tomography (PET) imaging biomarkers. Two new regularization terms was considered in a novel objective function. Discriminative learning was developed to jointly discover the internal relationship for AD/MCI classification. The effectiveness was demonstrated by comparing with the state-of-the-art methods for both clinical scores prediction and clinical label identification in [21].

Two robust pipelines, including extensive pre-processing modules and deep learning-based classifiers were presented in this proposed work [22]. Convolutional neural network architecture was used for feature extraction resulted in the highly accurate and reproducible predictive model. higher accuracy rate was achieved with LeNet and GoogleNet state of the art architecture for both the MRI and fMRI modalities .The progressive stages of Alzheimer's disease was predicted and the effects of aging in the normal brain was potentially classified.

A multi-layered neural network consisting of several auto encoders was trained using the selected feature subset in the training dataset. The softmax layer was added on the top of the stacked auto encoders (SAEs) for classification and then the trained network was evaluated with the labelled testing samples. Based on MR and PET ADNI data repository, proposed framework [23] outperformed the state-of-the art SVM-based method and other deep learning frameworks.
Multimodal Stacked Deep Polynomial Networks (MM-SDPN) algorithm [24] consists of two-stage Stacked Deep Polynomial Networks (SDPN) to fuse and learn feature representation from multimodal neuro-imaging data was suggested for AD diagnosis. Two SDPNs to learn high-level features of MRI and PET were first used, and then fed to another SDPN to fuse multimodal neuro-imaging information. Experimental results indicated that MM-SDPN is superior over the state-of-the-art multimodal feature learning based algorithms for AD diagnosis.

MRI data and neuropsychological test, MMSE were used as input to a multi-dimensional space to classify AD and it's prodromal stages-MCI including amnestic MCI (aMCI) and nonamnestic MCI (naMCI). SVM classifier was used and the results indicated that the brain atrophy was almost evenly seen on both sides of the brain for $\mathrm{AD}$ subjects, which was different from right-side dominance for aMCI and left-side dominance for naMCI. Furthermore, it was found that the hippocampal atrophy was most significant for aMCI, while Accumbens area and ventricle were most significant for naMCI [25].

Multimodal Imaging Brain Connectivity Analysis (MIBCA) functionalities [26] were used to study AD in a MRI and PET multimodal approach. Increased deposition of beta-amyloid was detected for the $\mathrm{AD}$ group. Major cortical atrophy particularly in the entorhinal cortex and temporal areas and the rise in the Mean Diffusivity in the hippocampus, amygdala and temporal areas were identified in the patients. It was found that as the disease progress, fiber connectivity was reduced for the intra-hemispherical connection.

A machine learning approach [27] for personalized and cost-effective diagnosis of $\mathrm{AD}$ was proposed. A classifier model was tailored for each patient, using locally weighted learning approach. The most informative or cost-effective biomarkers were computed. The $\mathrm{AD}$ versus controls and converters (cMCI) versus non-converters (nMCI) classification were done using the ADNI data. This approach was performed similarly to all data at once with the reduced number (and cost) of the biomarkers needed to achieve a confident diagnosis for each patient.

Diffusion tensor imaging (DTI) was one of the most effective MR tools for the investigation of the brain anatomy. In addition to the gray matter, histo-pathological studies indicated that white matter was also a good target for both the early diagnosis of $\mathrm{AD}$ and for monitoring disease progression. DTI measures have been used to determine significant differences between AD patients and controls, as well as to predict progression of disease in symptomatic non-demented individuals. Application of these findings in clinical practice remains to be confirmed [28].

MRI scan could inform that the hippocampus is smaller than it should be, but it couldn't identify the cause of the damage. The author proposed [29] that DTI scanning was more efficient to predict memory performance than measurement of hippocampus volume, especially in study participants over the age of 50.

DTI and MR Tractography had significant total direct

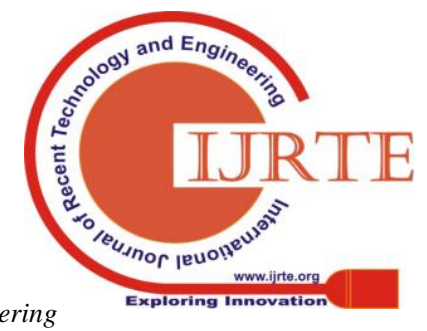


costs and resource utilization. No significant cost difference was seen between 1.5- and 3-Tesla MR systems for comparable number of encoding directions used. The total direct cost was found to increase just over $10 \%$ for both Tesla system with the increase in encoding directions from 15 to 32 . Post processing was the most expensive component of this test. Therefore priority should be given to the development of faster and robust post-processing software to decrease the overall total direct costs. Given the added value of DTI-MR tractography, dedicated reimbursement CPT codes must be developed to ensure the appropriate cost compensation [30].

Studies using Glucose hypo metabolism and gray matter atrophy as measures have shown memory impairment are associated with the degeneration of remote group of areas the mesial temporal lobe, Diencephalic structure such as mammillary bodies, anterior thalamus, and posterior cingulate. These sites were anatomically interconnected via a limbic - diencephalic network .DTI has recently confirmed degeneration of the white matter connections of the limbic diencephalic network in AD by way of a tract-based spatial statistics (TBSS) in [31].

Grey matter structures commonly implicated, including the hippocampus and posterior cingulate, had been identified as parts of a network vulnerable to early $\mathrm{AD}$ pathological changes. Diffusion changes in the grey matter 'nodes' of these networks could provide information that is complementary to the more studied changes in white matter [32].

Drawback of the DTI was found to be Limited spatial resolution of the actual image. As well as Tensor model was less informative in grey matter and region with low fractional anisotropy. Despite the relatively poor resolution of the actual images, DTI was powerful because it was found to be sensitive to molecular displacements on the order of $5-10 \mu \mathrm{m}$ in the usual tens of milliseconds given for water diffusion during the MRI measurements [33].

As DTI was found to depend on fast acquisition, it was known to suffer from severe distortion of image. This problem had been dealt in two ways, first post processing routines were used to reshape images to non-distorted form. Second was the use of alternative acquisition routes to significantly reduce these artefacts. Motions/pulsation artefacts - it was extremely sensitive to motion in the subject. Additional source of motion was found to be blood pulsation, which could cause small movements in the brain tissue. If two different white matter fiber systems were found to exist within the same voxel ("crossing fiber") the fractional anisotropy would be artificially reduced and would resemble grey matter. Fiber deviation because of the above certainty in fiber orientation estimation might be reduced. Q-ball imaging (QBI) and persistent angular structure (PAS/MRI) were found to be the solution for the above-mentioned limitations [34].

\section{B Retinal Image as a Biomarker}

Eye is considered as a non-invasive and inexpensive biomarker which shares many neural and vascular similarities to the brain. Fundus camera, Optical coherence tomography OCT, OCT angiography, Enhanced depth imaging optical coherence tomography. EDI-OCT and Polarization sensitive Optical Coherent Tomography (PS-OCT) were used to identify the eye based biomarkers. Sensitivity and diagnostic capacity for the disease were increased by the emerging technologies such as OCT angiography, EDI-OCT and PS-OCT.

Eye movements, Pupil Flash responses, Corneal nerve Imaging, Tear fluid proteomics, Crystalline lens $A \beta$ ligand signature, never fiber layer thinning polarization sensitive A $\beta$ signature, Blood Vessel changes, Choroidal thinning, Optic nerve pallor, Retinal $A \beta$ deposit and electrophysiological signature were the Ocular Biomarkers mentioned in this paper [35].

A sparsity coefficient was presented as a good marker of $\mathrm{AD}$ to differentiate AD EEG from both MCI and healthy controls. The thresholding wavelet approach was compared with the emerging method of compressive sensing. Results have shown the relationships between the compression ratio and the entropy measures of EEG complexity and considered the reduced complexity a hallmark of EEG in AD [41]. In another study, [42] EEG has been identified as an investigation tool and a potential biomarker for detecting dementia and classifying its severity by providing concise information about the brain activity and how it is affected by $\mathrm{AD}$ and vascular dementia $(\mathrm{VaD})$.

Instead of using a single EEG biomarker, information from multiple EEG biomarkers were combined to a diagnostic classification index in order to improve the accuracy of predicting conversion from MCI to AD within 2-year period. This work has shown [43] that multiple EEG biomarkers are mainly related to activity in the beta-frequency range $(13-30 \mathrm{~Hz})$ could predict conversion from MCI to AD with improved sensitivity and specificity when compared with the best individual biomarker. This approach could also be used to identify the optimal combination of biomarkers in other modalities.

High EEG upper/low alpha power ratio was found to be associated with cortical thinning and lower perfusion in the temporal-parietal lobe. Moreover, atrophy and lower perfusion rate were both considerably correlated with memory impairment in MCI subjects. Therefore the increase of EEG upper/low alpha frequency power ratio could be useful for identifying individuals at risk for progression to $\mathrm{AD}$ and found to be of value in the clinical context [44].

The left frontal cognitive ERP indicator could be a sensitive, simple, affordable, and non-invasive biomarker for early detection of aMCI [45].The authors of proposed work [46] believed that the biological complexity of the brain function and the physical "sum" effect of brain electrical fields on EEG recordings could make the understanding of EEG signals a very difficult task.

Even though EEG was affected by the problem of low spatial resolution when compared to other techniques (e.g., fMRI and PET), its high temporal resolution could makes it possible to highlight the mechanism of temporal synchronization of the cortical pyramidal neurons [47].

EEG signal was found to be cheap, non-invasive and anybody could take samples without the need of going to a medical facility each time.

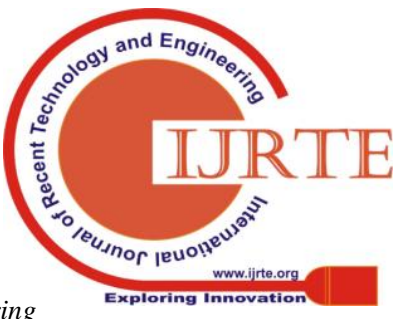
\& Sciences Publication 
But further research could be needed for EEG to be included in a clinical AD diagnosis [48].

\section{OLFACTORY DYSFUNCTION}

In the $\mathrm{AD}$ pathology, left hemisphere of $\mathrm{AD}$ patient had more degeneration than the right hemisphere. A test conducted on peanut and butter odour detection had proved that left nostril has greater decrement of odour detection sensitivity than right nostril of the AD patients [49]. This result has demonstrated the relationship of olfactory dysfunction with the $\mathrm{AD}$ pathology.

The relationship of Tangle density in the entorhinal cortex and CA1-subiculum with the odour identification was identified in the old age. Thus the neuro-fibrillary pathology is directly contributing to the olfactory dysfunction [50]. Brief Smell Identification Test (BSIT) analysed olfactory decline among the AD patients [51].

The University of Pennsylvania Smell Identification Test (UPSIT) and many other olfactory test were found to have high sensitivity, specificity and correlations. The modern peanut and butter odour identification test is economical and easy to administer. Even though several neurodegenerative and neuropsychiatric diseases were identified through the olfactory test, there was a lacking in the intervention literature. Therefore, these olfactory tests could be tested at least on first-degree relatives at risk for Alzheimer's disease [52].

\section{CONCLUSION}

The characteristics of non-invasive biomarkers such as MRI, fMRI, FDG-PET, MRS, DTI, Ocular Biomarkers, EEG signal and different olfactory tests are reviewed for the early diagnosis of AD. Positron tomography (PET) scanning combined with a brain-imaging agent called Pittsburgh Compound B can detect abnormal brain proteins, known as amyloid plaques. It is concluded that among the non-invasive biomarkers, DTI and EEG seem to be effective for the earliest sign detection of AD. But DTI is complex, time consuming and costly. The most promising biomarker for discriminating $\mathrm{AD}$ patients is MRI, while EEG gives the best results for MCI vs controls and MCI vs AD distinction. Low cost, affordability and portable features make EEG a promising technique to detect $\mathrm{AD}$ in the early stage. Olfactory test could be used along with other biomarkers for improved early diagnosis of $\mathrm{AD}$

\section{REFERENCES}

1. W. Thies, L. Bleiler, " Alzheimer's disease facts and figures," Alzheimer's \& Dementia, 2013; 9(2): 208-45

2. K. Strimbu, J. A. Tavel, "What are biomarkers? Current opinion in HIV and AIDS," 2010; 5(6): 463.

3. WHO International Programme on Chemical Safety Biomarkers in Risk http://www.inchem.org/documents/ehc/ehc/ehc222.htm. December 30, 2013.

4. Mingxia Liu, Daoqiang Zhang*, and Dinggang Shen*, Senior Member, IEEE, "Relationship Induced Multi-Template Learning for Diagnosis of Alzheimer's Disease and Mild Cognitive Impairment," IEEE TRANSACTIONS ON MEDICAL IMAGING, VOL. 35, NO. 6, pp. 1463 dysfunction symptoms occurred earlier than the cognitive Assessment: Validity and Validation. 2001.

5. Jin Liu, Min Li, Wei Lan, Fang-Xiang Wu, Yi Pan, and Jianxin Wang, "Classification of Alzheimer's Disease Using Whole Brain Hierarchical Network," IEEE/ACM TRANSACTIONS ON COMPUTATIONAL BIOLOGY AND BIOINFORMATICS, VOL. 14, NO. 8, 2015

6. Robin Wolz, Dong Ping Zhang, et al, "Multi-Method Analysis of MRI Images in Early Diagnostics of Alzheimer's Disease," PLoSONE .,www.plosone.org, Volume 6, Issue 10, e25446, 2011.

7. B. Al-Naami, N. Gharaibeh, and A. AlRazzaqKheshman, "Automated Detection of Alzheimer Disease Using Region Growing technique and Artificial Neural Network," International Science Index, Biomedical and Biological Engineering, Vol:7, No:5, pp. 204-208, 2013 waset.org/Publication/11271.

8. Luis Javier Herrera*, Ignacio Rojas, H. Pomares, A. Guillén, O Valenzuela, O. Baños, "Classification of MRI images for Alzheimer's disease detection," SocialCom/PASSAT/Big Data/EconCom/BioMedCom, pp. 846-851, 2013, IEEE.

9. Simon Duchesne*, Member, IEEE, Anna Caroli, et al. "MRI-Based Automated Computer Classification of Probable AD versus Normal Controls," IEEE TRANSACTIONS ON MEDICAL IMAGING, Vol 27, N0. 4, pp. 509-520, 2008.

10. Chenhui Hu, Xue Hua, Jun Ying, Paul M. Thompson, Georges E. Fakhri, Fellow, IEEE, and Quanzheng Li, "Localizing Sources of Brain Disease Progression with Network Diffusion Model," IEEE JOURNAL OF SELECTED TOPICS IN SIGNAL PROCESSING VOL. 10, NO. 7, pp. 1214-1225, 2016

11. R. Armañanzas, M. Iglesias, D. A. Morales and L. Alonso-Nanclares, "Voxel-Based Diagnosis of Alzheimer's Disease Using Classifier Ensembles," in IEEE Journal of Biomedical and Health Informatics, vol. 21, no. 3, pp. 778-784, May 2017. doi 10.1109/JBHI.2016.2538559

12. Tianhao Zhang*, Member, IEEE, and Christos Davatzikos, Senior Member, IEEE, "ODVBA: Optimally-Discriminative Voxel-Based Analysis," IEEE TRANSACTIONS ON MEDICAL IMAGING, VOL. 30, NO. 8, pp. 1441-1454, 2011.

13. Aoyan Dong*, Nicolas Honnorat, Member, IEEE, Bilwaj Gaonkar, and Christos Davatzikos, Fellow, IEEE, "CHIMERA: Clustering of Heterogeneous Disease Effects via Distribution Matching of Imaging Patterns," IEEE TRANSACTIONS ON MEDICAL IMAGING, VOL. 35, NO. 2, pp. 612-621, 2016.

14. Ching-Cheng Chuang, Pei-Ning Wang, et al, " Near-Infrared Brain Volumetric Imaging Method: A Monte Carlo Study," IEEE JOURNAL OF SELECTED TOPICS IN QUANTUM ELECTRONICS, VOL. 18 NO. 3, pp. 1122-1129, 2012

15. Jun Zhang, Yue Gao, Senior Member, IEEE, Yaozong Gao, Brent C Munsell, and Dinggang Shen*, Senior Member, IEEE, "Detecting Anatomical Landmarks for Fast Alzheimer's Disease Diagnosis," IEEE TRANSACTIONS ON MEDICAL IMAGING, VOL. 35, NO 12, pp. 2524-2533, 2016.

16. G. B. Frisoni, et al, "The clinical use of structural MRI in Alzheimer disease," Nat Rev Neurol. Author manuscript; pp.67-77, available in PMC 2011

17. Mingxia Liu, Daoqiang Zhang*, Ehsan Adeli, Member, IEEE, and Dinggang Shen*, Senior Member, IEEE,” Inherent Structure-Based Multiview Learning With Multitemplate Feature Representation for Alzheimer's Disease Diagnosis," IEEE TRANSACTIONS ON BIOMEDICAL ENGINEERING, VOL. 63, NO. 7,pp. 1473-1482, 2016

18. Biao Jie, Mingxia Liu, Jun Liu, Daoqiang Zhang*, and DinggangShen*,'Temporally Constrained Group Sparse Learning for Longitudinal Data Analysis in Alzheimer's Disease," IEEE TRANSACTIONS ON BIOMEDICAL ENGINEERING, VOL. 64 NO. 1, pp. 238-249, 2017

19. [L. Sørensen , C. Igel, N. Liv Hansen, M.Osler, M. Lauritzen, E. Rostrup, M. Nielsen, for the Alzheimer's Disease Neuroimaging Initiative and the Australian Imaging Biomarkers and Lifestyle Flagship Study of Ageing (2015), "Early detection of Alzheimer's disease using MRI hippocampal texture," Hum Brain Mapp, accepted which has been published in final form at DOI:10.1002/hbm.23091

20. E. M. Ali, A. F. Seddik, M. H. Haggag, "Automatic Detection and Classification of Alzheimer's Disease from MRI using TANNN," International Journal of Computer Applications (0975 - 8887) Volume 148 - No.9 pp. 30-34, 2016

21. Baiying Lei, Member, IEEE, Peng Yang, Tianfu Wang, Siping Chen, and Dong $\mathrm{Ni}$

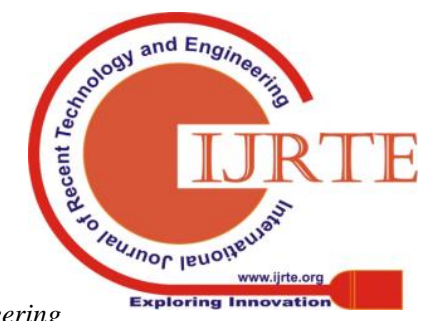


Member, IEEE, "Relational-Regularized Discriminative Sparse Learning for Alzheimer's Disease Diagnosis," IEEE TRANSACTIONS ON CYBERNETICS, VOL. 47, NO. 4, pp. 1102-1113, 2017.

22. Saman Sarraf, GhassemTofighi, for the Alzheimer's Disease Neuroimaging Initiative,'DeepAD: Alzheimer's Disease Classification via Deep Convolutional Neural Networks using MRI and fMRI," bioRxiv preprint first posted online Aug. 21, 2016; doi: http://dx.doi.org/10.1101/070441.

23. Siqi Liu*, Student Member, IEEE, et al, "Multimodal Neuroimaging Feature Learning for Multiclass Diagnosis of Alzheimer's Disease," IEEE TRANSACTIONS ON BIOMEDICAL ENGINEERING, VOL. 62, NO. 4,,pp. 1132-1140, 2015

24. J. Shi; X. Zheng; Y. Li; Q. Zhang; S. Ying, "Multimodal Neuroimaging Feature Learning with Multimodal Stacked Deep Polynomial Networks for Diagnosis of Alzheimer's," Disease in IEEE Journal of Biomedical and Health Informatics, vol. PP, no.99, pp.1-1. doi: 10.1109/JBHI.2017.2655720

25. Qi Zhou, Mohammed Goryawala, et al, "An Optimal Decisional Space for the Classification of Alzheimer's Disease and Mild Cognitive Impairment," IEEE TRANSACTIONS ON BIOMEDICAL ENGINEERING, VOL. 61, NO. 8, pp. 2245-2253,2014

26. Rajesh, M., and J. M. Gnanasekar. "Path Observation Based Physical Routing Protocol for Wireless Ad Hoc Networks." Wireless Personal Communications 97.1 (2017): 1267-1289.

27. André Santos Ribeiro, Luís Miguel Lacerda, Nuno André da Silva and Hugo Alexandre Ferreira for the Alzheimer's Disease Neuroimaging Initiative, "Multimodal Imaging of Brain Connectivity Using the MIBCA Toolbox: Preliminary Application to Alzheimer's Disease," IEEE TRANSACTIONS ON NUCLEAR SCIENCE, VOL. 62, NO 3,pp. 604-611, 2015.

28. Javier Escudero*, Member, IEEE, Emmanuel Ifeachor, Member, IEEE, et al, "Machine Learning-Based Method for Personalized and Cost-Effective Detection of Alzheimer's Disease," IEEE TRANSACTIONS ON BIOMEDICAL ENGINEERING, VOL. 60, NO. 1, pp. 164-168, 2013.

29. N. Nithiyanandam, K. Venkatesh, M. Rajesh, Transfer The Levels Of The Monitored Carbon, Nitrogen Gases From The Industries, International Journal of Recent Technology and Engineering, Volume-7 Issue-6S3 April, 2019.

30. Sivanesh Kumar, A., Brittoraj, S., Rajesh, M., Implementation of RFID with internet of things, Journal of Recent Technology and Engineering, Volume-7 Issue-6S3 April, 2019.

31. Rajesh, M., Sairam, R., Big data and health care system using mlearningJournal of Recent Technology and Engineering, Volume-7 Issue-6S3 April, 2019.

\section{AUTHORS PROFILE}

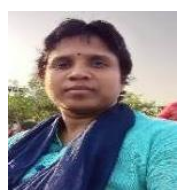

Ramya.M received B.E. Degree in Electronics and Communication Engineering from St. Peters Engineering College, Madras University, Tamil Nadu, India in May 2002. She worked as a Senior lecturer in ECE department, S.A Engineering college from 2002 to 2007. She received M.E degree in Embedded System and Technologies from S.A. Engineering college, Anna University, Tamil Nadu in 2009. She is currently working towards the Ph.D. degree at the research center of Jerusalem college of Engineering, Anna University, TamilNadu, India. Her research interest includes Neuroimaging and Biomarkers.

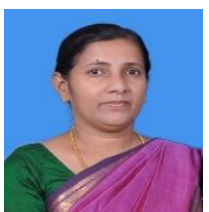

Marykutty Cyriac received her B.Tech in Electronics and Communication Engineering (1985) from University of Kerala, Master's degree in Applied Electronics (2004) from Anna University, Chennai and Ph.D. degree in Image Processing (2014) from Anna University, Chennai. Previously she has worked with Sanmar group of companies in their computer manufacturing division. Currently she is a Professor in the Department of Electronics and Communication Engineering, Jerusalem College of Engineering, Chennai. She has published papers in international journals and presented papers in national and international conferences. She is a life member of ISTE and BMESI. Her areas of interests are medical image processing, image compression and pattern recognition.

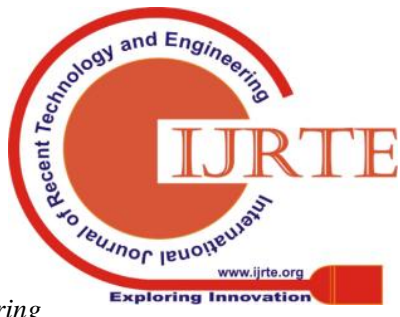

